\title{
ERRATUM
}

\section{Relative manifold based semi-supervised dimensionality reduction}

\author{
Xianfa CAI $(\bowtie)^{1,2,3}$, Guihua WEN ${ }^{2}$, Jia WEI ${ }^{2}$, Zhiwen YU ${ }^{2}$ \\ 1 School of Medical Information Engineering, Guangdong Pharmaceutical University, Guangzhou 510006, China \\ 2 School of Computer Science and Engineering, South China University of Technology, Guangzhou 510006, China \\ 3 Shenzhen Key Laboratory of High Performance Data Mining, Shenzhen 518055, China
}

(c) Higher Education Press and Springer-Verlag Berlin Heidelberg 2015

Erratum to: Front. Comput. Sci., 2014, 8(6): 923-932. DOI 10.1007/s11704-014-3193-8

Figure 8 of this article shows YaleB and CMU PIE with incorrect legend titles: YaleB $(\operatorname{Tr}=1900, \mathrm{Te}=514, \mathrm{NOC}=100)$ should be YaleB $(\mathrm{Tr}=1900, \mathrm{Te}=514, \mathrm{~d}=100)$ (Fig. 8(a)); TIE $(\mathrm{Tr}=1200, \mathrm{Te}=2880, \mathrm{~d}=100)$ should be $\mathrm{PIE}(\mathrm{Tr}=1200, \mathrm{Te}=2880, \mathrm{~d}=100)$ (Fig. 8(b)).

In Fig. 9, the legend keys and the legend texts are mismatched. The correct figure is illustrated as follows.
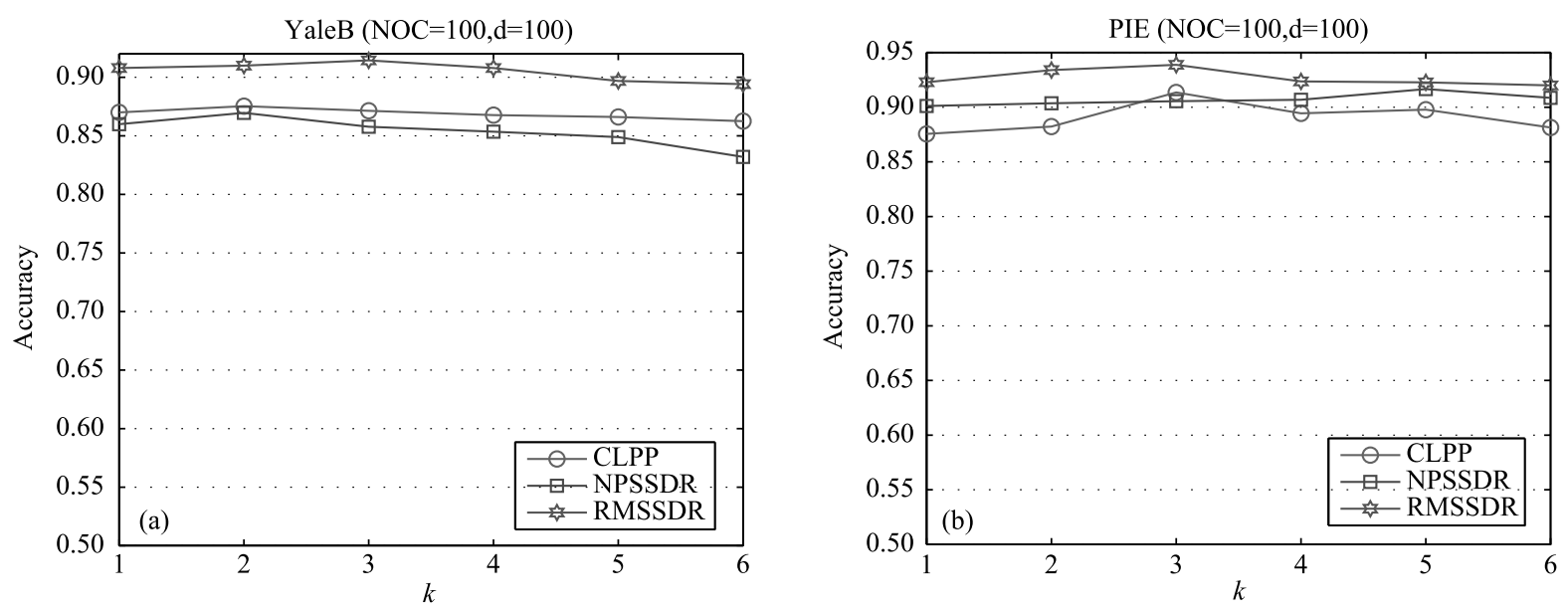

Fig. 9 Classification accuracies of YaleB and CMU PIE on the different $k$ s. (a) YaleB; (b) PIE 